\title{
Pharmacists Prescribing: A Professional Re-Stratification or a Back to the Future Scenario?
}

\begin{abstract}
Substantial effort has been applied to researching government initiatives aimed at increasing efficiency and cost effectiveness within the UK healthcare system, particularly in terms of medical practice. However, little attention has been paid to the impact of these on the boundaries of authority within the setting of hospital pharmacies. This paper utilises the sociology of the professions to analyse and report on hospital pharmacist prescribing in Scotland. The findings indicate a reduction in the authority of the medical profession and that medical practice can be subjected to rationalisation in the same way as other occupations which is of sociological importance.
\end{abstract}

Keywords: hospital prescribing, medical jurisdiction, rationalisation, professional status

\section{Introduction}

Over the last four decades, financial constraints and reforms of the UK's National Health Service (NHS), driven by managerial and accounting logics aimed at controlling healthcare expenditure, have put considerable pressure on the medical profession to reconfigure professional roles and competencies (Martin, Currie \& Finn, 2009; Jackson et al., 2014). Indeed, a persistent theme of NHS reform has been an attempt to improve efficiency and value for money through workforce reconfigurations that maximise the specialist expertise of professions allied to medicine, such as pharmacy (Audit Commission, 1994; DOH, 2000a, 2000b, 2005 \& 2008). In the year 2000, the Department of Health (DOH) asserted that 'NHS staff, at every level, are the key to reform ... [and it] is about working smarter to make maximum use of the talents of all the NHS workforce' (DOH, 2000a, p.82). As a result of this initiative, pharmacists in the hospital setting have become integral actors within healthcare teams, increasingly taking on more authoritative roles and clinical responsibilities including greater authority over prescribing practice (DOH, 2003, 2004, $2007 \& 2008$; Weiss \& Sutton, 2009). Theorists of the profession argue that such role reconfigurations can enhance the professional status of one group (e.g. pharmacists), but will inevitably have a deprofessionalisation effect on another (e.g. clinicians), which is unlikely to go uncontested (Britton, 2001; Ritzer \& Walcak, 1982). Indeed, the sociology of the professions literature reveals that the medical profession tends to vigorously defend its jurisdiction and professional boundaries (Abbott, 1988).

A defining feature of a professional is their level of autonomy (Abbott, 1988), and prescribing has been identified as one of the key hallmarks of the medical profession and as an aspect of their professional jurisdiction (Larson, 1977). Indeed, within the wider structures of society, the monopoly over prescribing has been an indicator of the medical profession's clinical autonomy and power, with full jurisdiction for prescribing being the held within the domain of medicine (Freidson, 1970; Mesler, 1991; Buckley, Grime \& Blenkinsopp, 2006). Pharmacist prescribing, therefore, represents a shift in this jurisdiction, which has the potential to alter both professional status and interprofessional relationships. While the expansion of non-medical prescribing through pharmacist and nurse prescribing ${ }^{i}$ may not yet have impacted significantly on overall medical control, it has created interprofessional boundary tensions, struggles for professional autonomy, (Freidson, 1994; Connelly, 2005; Abraham, 2009; Weiss \& Sutton, 
2009) and has provided the conditions for a re-stratification within healthcare professions (Martin, Currie \& Finn, 2009). However, little attention has been given to the impact of attempts to improve efficiency and value for money through workforce reconfigurations such as pharmacist prescribing that maximise specialist expertise. This is surprising given that medicines constitute the principle non-surgical intervention in healthcare provision. Likewise, sociological investigation into the social context and interprofessional relationships of healthcare professionals has tended to overlook pharmacists, favouring the medical profession as the primary area of interest (Anderson, 2005). Such work has also tended to overlook the historical position of pharmacy as a strong profession, portraying it instead as a marginal or quasi-profession.

Some studies have demonstrated that the medical profession have been responsive to the introduction of managerial logics and workforce reconfigurations, but these have tended to be in areas that have little impact on their clinical autonomy and jurisdiction (Allan, 2001). On the other hand, a much larger body of literature shows considerable resistance from the medical profession against managerial intrusions that have the potential to affect their jurisdictional boundaries and professional autonomy (Martin, Currie \& Finn, 2009; Drennan et al., 2017). However, the impact of managerial logics and workforce reconfiguration on prescribing practice in the hospital setting has previously not been examined. The aim of this study is to contribute to filling this gap by investigating the changing status of pharmacists as professionals and its evolving interprofessional relationship with the medical profession following managerial reforms that extend prescribing authority into their function.

The paper begins with a brief outline of the historical position of pharmacy as a profession and the development of hospital pharmacy today to demonstrate the (re-)professionalisation of pharmacists. Following this the research approach and data analysis are put forward. The study findings are then presented. Through an interpretative framework the study findings are evaluated. Drawing on the accounts of pharmacists and clinicians highlights a change in perceptions of the professional status of both groups due to workforce reconfigurations arising from managerial logics being imposed and illuminates resultant professional tensions. Using the sociology of the professions, the analysis contributes to the understanding of the development of pharmacy as a profession and its evolving interprofessional relationship with the medical profession. The final section presents the study discussion and conclusion, its limitations and scope for further research in this area.

\section{The (Re)Professionalisation of Pharmacy Through Healthcare Reform}

When considering the status of pharmacy as a profession, it is important to note that pharmacy is one of the oldest healing professions and was once held in higher regard. The history of pharmacy and the making of medicines can be traced back over 4000 years (Court, 2005), during which time great shifts in both medical practice and professional boundaries (see Figure 1) have taken place (Anderson, 2005). 


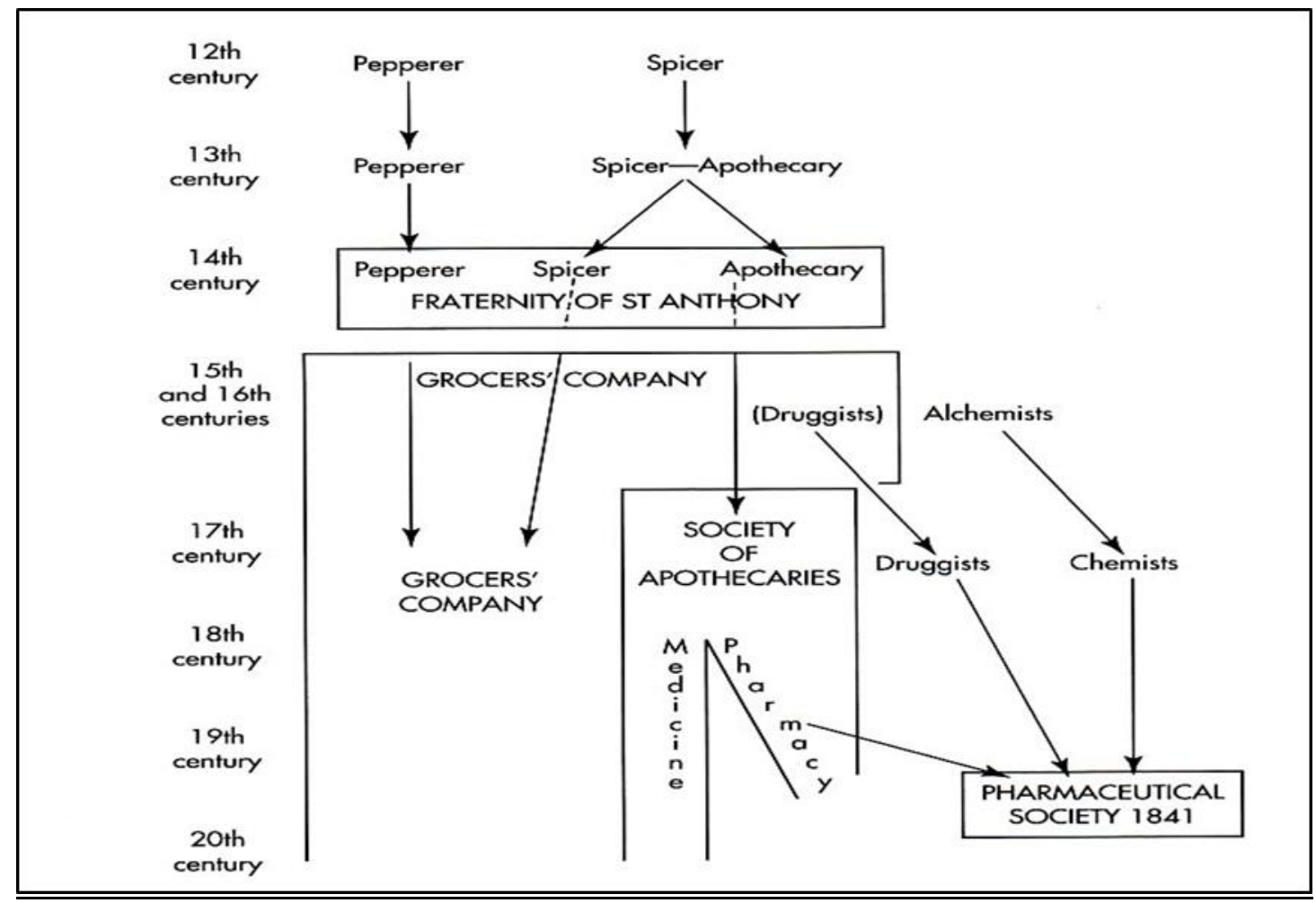

Figure 1: The Evolution of Pharmacy

(Anderson, 2005: p.12)

When reviewing the early history of pharmacy and medicine, the two professions are practically inseparable with Spicer-Apothecaries (an early term for pharmacists) not only dispensing medicines but also prescribing them (Trease, 1964); a situation which persisted in many parts of the world including the British Isles until the end of the $17^{\text {th }}$ century (Homan, 2005). At this time pharmacy enjoyed a status comparable to medicine through its understanding and control over an exclusive field of specialised knowledge concerning the compounding and dispensing of medicines. It also had control over remuneration for its services, another feature which is attributed to occupational groups that are identified as professions.

The history of pharmacy portrays the historical relationship between Apothecaries and Physicians as one of ongoing conflict and competition as a result of the skills and service overlap of the two professions (Barrett, 1905; Poynter, 1965). The right to prescribe was particularly contentious and came to a head in Britain in 1704 in a legal battle in the House of Lords (the Rose Case) the outcome of which a ruling that apothecaries could both prescribe and dispense medicines (The Worshipful Society of Apothecaries). It was shortly after this period that the Society of Apothecaries evolved into general medical practitioners and the pharmacy profession grew out of the Association of Chemists and Druggists, which led to the formation of the Pharmaceutical Society in 1841. Later the same year they were granted a Royal Charter representing the beginnings of modern pharmacy in the UK. From then onward, moves were made to increase the education of pharmacists with statutory qualifications being imposed and reinforced through the pharmacy education Act in 1886 (Anderson, 2001). Expansion of formal education continued with a degree qualification appearing in the UK in 1924. By 1948 a three-year honours degree had been introduced at the University of London, thus laying the foundations of the formal pharmacy education system that is in place today. 
Alongside these changes, the expansion of the pharmaceutical manufacturing industry also had a significant impact on the pharmacy profession. As research within the large drugs companies increased, the significance of the traditional compounding role of pharmacists diminished. Further, as propriety drugs became widely available at reduced prices, pharmacy's role in production gradually became negligible. As a consequence of these technological and economic changes, pharmacists found themselves being viewed as over trained and qualified for their role and became labelled as a quasi or marginal occupation (Eaton \& Webb, 1979). These changes also contributed to the structural fragmentation of the occupational group with two principal sectors emerging: private business (retail) and medical (hospital-based) pharmacy (Turner, 1995), which in turn are fragmented both vertically and horizontally in the form of hospital pharmacists, administrators, industrial chemists and retail pharmacists. Such fragmentation made the continued development of a common professional code of practice difficult to achieve or sustain.

The social, economic and political contexts in which pharmacy has existed have largely developed and shaped the role and practice of pharmacy as it is known today (Anderson, 2005). UK hospital pharmacists, in particular, have responded to the existential threat posed by falling status, by developing services and clinical skills that strongly supported the activities of the medical profession and ensured their continued relevance to the health service (Anderson, 2005). Indeed, hospital pharmacy has undergone significant development since the 1970s; moving from the traditional dispensing role into a clinically oriented service that includes medicines management programmes and the writing of discharge prescriptions (Buckley, Grime \& Blenkinsopp, 2006). The Royal Pharmaceutical Society of Great Britain (RSPGB) has actively engaged in professional developments that push the boundaries of the pharmacist's role beyond compounding and dispensing into clinical activities and developed strategies, training and services for Pharmacy in a New Age (Holloway, Jewson \& Mason, 1986; RPSGB, 1986, 2002, 2009).

Successive governments also have recognised the potential of pharmacists to contribute to more efficient and effective medicines management (DOH,1988). Greater involvement of pharmacists in prescribing decisions and practice was deemed essential to the control of prescribing costs and ensuring patient safety (Audit Commission 1994), while the Department of Health (DOH 1999, p8) observed that 'pharmacists in all sectors, working as part of the clinical team, have an important role in ensuring consistent standards of care across the NHS'. In this vein, the NHS Executive initiated reforms which have facilitated an expanded role for pharmacists and task redistribution such as the promotion of advisory and healthcare services and clinical pharmacy services to support efficient and cost-effective prescribing ${ }^{\text {ii }}$, and clinical governance (NHS Executive, 1999). For example, an Area Prescribing and Medicines Management Committee ${ }^{\mathrm{iii}}$ was established that had the remit to develop and co-ordinate strategic medicines management by providing consistent, evidence-based advice for the improvement and cost-effectiveness of prescribing across the primary/secondary care interface. Such committees worked in conjunction with the National Institute for Health and Care Excellence (NICE) and its Scottish equivalents, the Scottish Intercollegiate Guidelines Network and the Scottish Medicines Consortium ${ }^{\text {iv }}$, to develop prescribing guidelines and protocols ${ }^{\mathrm{v}}$ for the safe and cost-effective use of medicines (Jackson et.al., 2014).

The (1999) DOH Report on the Review of Prescribing and Supply of Medicines also recognised the constraints that had been placed on the professional practice of pharmacy and the waste of highly trained human resources that resulted. Its recommendations urged the expansion of 
supplementary prescribing to include pharmacists for both the betterment of the health service and the easing of professional frustration:

'it is desirable that highly trained health professionals should be able to use their full range of skills in the interests of better patient care, the efficient use of resources and enhanced job satisfaction (DOH, 1999, p.19).

As the new millennium ensued, a barrage of reports from the $\mathrm{DOH}$ and other agencies hammered home the need for change. The NHS Improvement Plan, observed that 'unnecessary boundaries exist between the professions which hold back staff from fulfilling their true potential' (DOH, 2000a. p.27) while Pharmacy in the Future bemoaned that 'pharmacists are highly qualified professionals, whose skills the NHS has been under-utilising for too long' (DOH, 2000b, p.9). In 2001 the Audit Commission's A Spoonful of Sugar argued that pharmacy services need to be seen as a 'core clinical function not a technical support service' (p.44). A Vision for Pharmacy in the New NHS emphatically stated that 'Pharmacists are probably the biggest untapped resource for health improvement' (DOH, 2003, p.7) and that the 'value of their role within the NHS cannot be overestimated' (DOH, 2003, p.23). Thus, with drug treatments being one of the largest expenditures in the NHS, pharmacists were increasingly seen as key players in government drives to push down expenditure, optimise the utilisation of scare resources, and promote efficiency and value for money through improving managerial capacity, establishing collaborative networks and the redistribution of critical roles such as prescribing. ${ }^{\text {vi }}$ These messages were strongly supported from within the pharmacy profession when the Pharmacists Prescribing Task Group published a report that was closely aligned to the government rhetoric (RPSGB, 2002).

Cumulatively, the recommendations outlined above paved the way to a supplementary prescribing role being opened to pharmacists in 2004 (Scottish Government, 2004). The apparent success of pharmacist supplementary prescribing in improving cost effectiveness and patient medicines management then provided further support for the development of independent prescribing rights for pharmacists (Cooper et al., 2008), which led to an amendment to the Statutory Instruments 2006, No 915, Medicines Order to permit pharmacists independent prescribing rights (except for three specific controlled drugs for the treatment of addiction) for any condition within their clinical competence (Statutory Instrument, 2006).

The core theme within the reforms outlined above are centred on achieving efficient and costeffective medicines management through interprofessional working and task redistribution that maximises specialist expertise. The nature of these changes when taken together, are considered by some social scientists to be a (re)professionalising opportunity for pharmacy and a direct challenge to the autonomy and professional standing of the medical profession through the imposition of bureaucratic rule (McKinlay, 1982; Edmunds \& Calnan, 2001; McDonald et al., 2010). While the medical profession holds the same ideal as government in terms of wishing to create and provide an outstanding healthcare system, the perspectives contained within the reforms such as prescribing guidelines and protocols that impose autonomy constraints and task redistributions that affect their professional boundary are unlikely to go uncontested (Britton, 2001; Abraham, 2009; Weiss \& Sutton, 2009).

\section{Professional Autonomy and Managerial Logics}

The changes highlighted above will now be considered through the application of the sociology of the professions. In the study of the concept of 'profession', autonomy has been identified as 
being a central component (Harrison \& McDonald, (2003). Indeed, the principle of clinical autonomy has permeated the management and organisation of the NHS since its inception and the ability to prescribe has long been seen as a marker of the professional power and autonomy of the medical profession (Abbott, 1988; Mesler, 1991). The proximity between clinicians and the substance prescribed is encapsulated by the use of the word medicine which describes both the substance and the profession (Britten, 2001). However, within the deprofessionalisation thesis, it is argued that the medical profession is losing its cultural authority in terms of prestige, trust and autonomy as a result of political changes, workforce reconfigurations and increased awareness and knowledge of the patients which it serves (Harding \& Taylor, 1997; Haug, 1988).

Arguably, the specialist pharmacological training that pharmacists undertake places pharmacists as society's real experts on medicines; providing and ensuring their safe and effective use through their information and advice (Britton, 2001). But, as discussed above, pharmacy as a health care function has in the past been termed a marginal or quasi-profession due to its apparent inability to promote and control its existence. Traditional professional boundaries and territoriality militated against a closer working relationship between pharmacists and clinicians, contributing to this marginalisation (Claesson, 1989). Furthermore, Jamous \& Peloille (1970) with their technicality/indetermination ratio ${ }^{\text {vii }}$, argue that a significant problem for pharmacy lies within its precise and systematic knowledge base, which is founded on exact science that does not allow for a wide range of interpretation and thus leaves little scope for judgement or hermeneutics. Finally, pharmacy as a profession had not fully developed a patient counselling role in its own right. Rather this has been mediated through the medical profession. The result is an occupational grouping with a highly developed science in the form of pharmacological knowledge, but under-developed interpretative skills in relation to patient welfare (Denzin \& Mettlin, 1968), thus reinforcing the perception of pharmacy as a marginal or quasi-profession.

Hospital pharmacy as a distinct sub-grouping of general pharmacy, has, on the other hand, developed clinically orientated services and a skill set that complements and overlaps the medical profession in a way that challenges the perception of it as a marginal or quasi-profession. The potential for this was spotted in the early days of the professionalisation literature when Freidson and Lorber (1972) noted that the services of pharmacy were essential to the practice of medicine and contended that developments in pharmaceutical services and pharmacist prescribing could potentially threaten the medical profession's position and lead to inter-professional conflict. Eaton and Webb (1979) countered that the expansion of clinical pharmacy activity into prescribing practice would not necessarily offer much threat in terms of boundary encroachment if clinicians delegated some of the tasks involved in prescribing, such as drug therapy monitoring and counselling to clinical pharmacists. They further argued that such an expansion did not suggest that the dominance of medicine would be threatened but rather that it would indicate a renegotiation of order within a limited, predefined area in which clinicians retained control over the patient management programme. However, later writers such as Holloway, Jewson \& Mason (1986), Elston, (1993) and Weiss and Fitzpatrick (1997) recognised that pharmacists increased involvement in clinical activities such as drug history taking, patient counselling and prescribing did appear to be boundary encroachment and could be regarded as a professionalising strategy and a means to enhance their professional status. Freidson, (1986, 1988) further contended that the medical profession would be driven to circumscribe the activities of pharmacists in order to sustain the medical profession's dominant position. 
From this standpoint, it makes sense to examine the effects of managerial logics and workforce reconfigurations and their potential impact on professional autonomy. In particular, if managerial logics and workforce reconfigurations are viewed as a form of bureaucratic rule, this then raises questions with regard to their potential to undermine of the status of the medical profession (Allsop, 2006; Harrison \& Checkland, (2009). Indeed, Abernethy and Vagnoni (2004) argue that managerial logics and workforce reconfigurations challenge the power and autonomy of clinicians and their relationship with other healthcare professionals and society. Gray and Harrison (2004), further contend that managerial logics, workforce reconfigurations, and other systematizations of medical practice can ostensibly be regarded as a government attempt to reconfigure aspects of clinical practice into a set of scientific or technical rules which through their political legitimacy suppress contestation. Such initiatives, when taken together, are argued to represent a new form of bureaucracy that is characterised by policies and formal rules that increase the level of surveillance and decrease professional autonomy (Harrison, 2003).

It is the effect of the outputs of these policies and rules that are examined here. The problem can be seen as one of the weakening of a jusrisdictional boundary; in this case the boundary between the medical and pharmaceutical domains and the resultant migration of pharmacists into that domain. Abbot (1988, p. 89) has argued that 'many or most jurisdictions are uniquely held', but the introduction of policy instruments, guidelines and formularies and the use of interdisciplinary collaborative groups (e.g. NICE and SIGN) can be seen as the introduction of 'boundary objects' that weaken and mediate the jurisdictional boundary and create boundary bridges (see for example Kislov, 2014). Reflecting on the work of Jamous and Peloille (1970) these boundary objects do their work by tehnicising and codifying aspects of prescribing practice in a way that shifts them from the indeterminate domain of medical (and pharmacological) knowledge. Figure 2 (below) provides a model of this interaction:

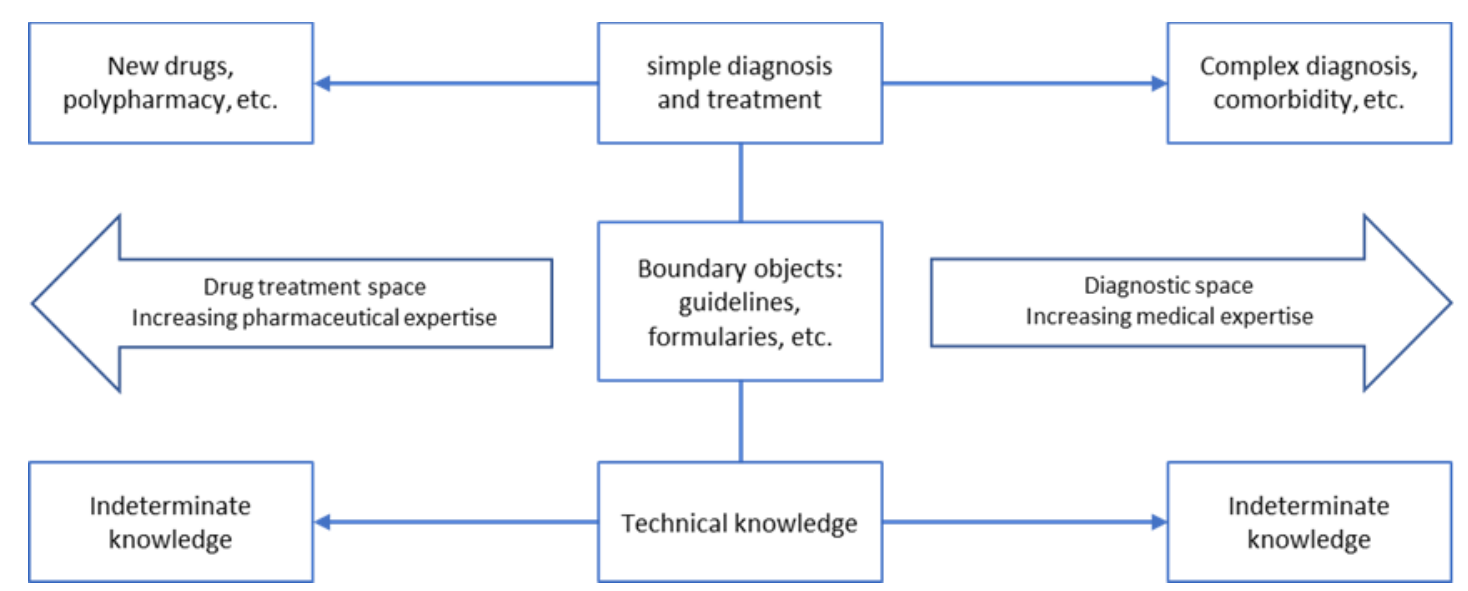

Figure 2: A model of the jurisdictional boundary between Medicine and Pharmacy

From figure 2, each profession has an abstract, indeterminate field of knowledge with an overlap at the point of diagnosis/treatment. The training of each group ensures that each has some knowledge of the other field, with clinicians traditionally claiming sufficient knowledge over the pharmacological field to have primacy over prescribing, a claim that pharmacy found hard to resist due to their more limited knowledge of diagnosis. But the introduction of clinical guidelines and formularies has reduced the clinicians' hold over some areas of prescribing practice and Jackson et al., (2014) showed that many, particularly junior, clinicians felt that 
their clinical autonomy (jurisdiction) had been eroded. It is from this standpoint that the development of clinical pharmacy is examined.

\section{Sources and Research Method}

To explore the above issues, this paper reports on a case study of hospital pharmacists prescribing in Scotland. The study seeks to locate itself within the management and the control of medicines in secondary care and specifically examines the effect of the expansion of prescribing into the clinical pharmacy role on the interprofessional relationships and status of pharmacy and the medical profession. In particular, there is critical reference to the foundation of a profession and role boundaries. As outlined above, the right to prescribing has been a hallmark and indicator of the dominance of the medical profession (Abbott, 1988; Mesler, 1991; MacDonald, 1995). The introduction of pharmacist prescribing supported by external forces and organisational structural changes represents a challenge to this professional jurisdiction (Abbott, 1988, p.91-6). The central objective of this paper is, therefore, to investigate the impact of this challenge to prescribing jurisdiction on the interprofessional relationships between hospital clinicians and pharmacists.

The research approach adopted for this study was a qualitative paradigm as it enables the examination of structural processes and participants in their daily routines (Schultz, 1967), and facilitates an understanding of actions in terms of how and why they occur (Cassel \& Symon, 1994). This approach also provides the flexibility to investigate issues associated with the phenomena under study from the participants perspective (Blaikie, 2000). The data was gathered over the period 2011-2016 from three NHS Trusts within the central belt of Scotland. All three sites provide a comprehensive range of health services including acute and chronic hospital services covering a population between $300,000-1.2$ million with an average staffing of between 8,000-44,000. Each site also provides hospital pharmacy services. The sites were chosen according to accessibility. Ethical approval for the study was granted from the relevant NHS Trusts and confidentiality was guaranteed, therefore, no participants or locations are specifically identified. The data was gathered from thirty-eight participants using semistructured interviews which were designed to tease out the experience, attitudes and sensitivities of pharmacists prescribing on their perception of professional status and interprofessional relationships. The interview questions were guided by issues identified in prior literature and previous studies conducted by the researcher. The study participants included three clinical directors, six senior clinicians, seven clinicians, three directorate (clinical) pharmacists, four pharmacy managers, nine clinical pharmacists and six pharmacists. Appendix 1 provides details on the participants codes, site number and the duration of the interviews. Participation in the interviews was entirely on a voluntary basis. Notes were taken by the researcher during the interviews and, where permission was granted, the interviews were recorded and transcribed.

Following the first batch of six interviews, an initial data analysis was conducted in order that any necessary adjustments to the semi structured interview questions could be made, but no changes were deemed necessary to the original interview questions. The interview data was examined several times to enable familiarity and identification of emergent themes inductively (Paterson et al., 2016). To conceptualise the data a simple coding procedure was applied. An iterative approach was adopted for the data organising that involved the repeated comparison of the data themes to identify similarities and differences which were grouped into appropriate descriptive categories (Thomas \& Harden, 2007). The recurring themes centred around what 
constitutes prescribing, prescribing issues and developments, professional status and interprofessional relations.

An interpretivist approach was adopted for the data analysis. The interpretative approach seeks to ascertain why actors behave in certain ways by unearthing the largely tacit, mutual knowledge, symbolic meanings, motives and rules which govern their behaviour (Corbin \& Strauss, 2008; Blaikie, 2000, p.115). Such an approach offers the possibility to generate an understanding of changes in practice from the actor's perspective and facilitates analysis of how this is woven into a wider social framework (Chua, 1986). The primary objective was to identify issues and developments that are perceived by the study participants to have specifically affected their professional status and interprofessional relationships.

As with other qualitative studies, this study has its limitations which stem from its sample size and being restricted to one geographical location, which does not permit generalisations (Corbin \& Strauss, 2008) on the full impact of managerial logics and workforce reconfiguration on the interprofessional relationship and status of the two professions under examination. Additionally, the word constraints of the article restricted the scope of background material and use of participant views and opinions. As such, extreme care was taken to ensure that the most important relevant sources were included in the background material. Likewise, the core reflections and exact meaning of the study participants are presented in verbatim. The author accepts responsibility for any deficiencies that may appear in this respect.

As one of the first studies to examine the impact of managerial logics and workforce reconfiguration on hospital pharmacist prescribing, the study provides a contribution to the medicine's management and the sociology of the profession's literature demonstrating that medical practice can be subjected to rationalisation in the same way as other occupations. Furthermore, it sheds light on important issues regarding the interprofessional relationship between pharmacists and clinicians and opens up opportunities for more research in this area.

\section{Empirical Results and Reflections}

In this section the perceptions and responses of the study participants to pharmacist prescribing are put forward. The findings are laid out in two parts. The first part offers insights into the pharmacist's perspective on prescribing, professional judgement and inter-professional working. The second part introduces the views and opinions of clinicians on pharmacists prescribing, professional knowledge and the impact on interprofessional working and status.

\section{Pharmacist Perspective: Prescribing}

As previously discussed, within the study of the professions and the wider structure of society, the ability to prescribe has traditionally been used as an indicator of the professional power and autonomy of the medical profession (Abbott, 1988; Mesler, 1991). Indeed, full jurisdiction over the right to prescribe has been a defining feature of the medical profession. The Oxford Dictionary definition of prescribe is 'to advise or order the use of a medicine with directions for the manner of using it'. A series of events precedes any prescribing decisions. These involve, taking note of the patient's clinical history, making a clinical diagnosis through the application of a clinical examination which then leads to a decision on which therapeutic treatment is most appropriate. Thus, the prescription is determined by the drawing on and application of detailed scientific knowledge and experience as appropriate to the individual patient circumstances. 
The interviews opened with a discussion of pharmacist's perceptions of prescribing and this new role which led to some interesting views and opinions as to what constitutes prescribing. The evidence presented illustrates that defining prescribing within the hospital setting is not clear cut in the minds of pharmacists or perceived as a significant change to aspects of their practice.

'Well it depends on what you consider to be prescribing. We [clinical pharmacists] attend the clinical rounds and discuss the patient's medications in relation to their medical condition and are actively involved in determining their medications, suggesting the most appropriate medications and checking the interactions and such like so through our input into these rounds we were in a sense making prescribing decisions it's just that they [the clinicians] were putting pen to paper and not us'. (CP3S1).

A further point that was emphasised was that formal prescriptions (as used in primary care) are not utilised within the hospital setting: 'We don't use prescriptions like the ones issued by the GPs we have patient record cards.' (CP1S1). These charts are an authorisation to supply and to administer medication which was argued is not necessarily a prescription, particularly if the medication on the chart is medication that was prescribed by a GP, then it is rather considered a transcription. Additionally, it was debated whether or not an omission in the patient's drug chart on admission which was identified and corrected by the pharmacist constituted a prescribing decision.

'I had a patient with a heart condition who was on statins and aspirin $75 \mathrm{mg}$ come onto the ward, but the aspirin was left off his chart, so I added it on so would that count as prescribing?' (CP9S3).

With regards to prescribing, interestingly more than half of the pharmacist's respondents did not recognise that this was a major change to their activity compared to previous practice, observing that 'we've always made prescribing recommendations and added things to patient charts if needed, it's just that now it's more formal'. (CP2S1).

\section{Professional Judgement, Quality and Safety}

One key feature identified in the role of the prescriber is the demonstration and application of professional judgement. Reflecting on the historical role of pharmacists it can be argued that pharmacists have held such attributes all along. Indeed, pharmacists today through the principles of safe and appropriate production of medicine administration charts laid down by the RPSGB (2009) and the General Pharmaceutical Council (2018) still hold the legal right to refuse the supply of a substance if their professional judgement determines that it is inappropriate or in conflict with the patient's other medication regime: 'we can refuse to supply a medicine if we believe it inappropriate'. (PM3S2). Several respondents held the view that given the need for safe prescribing practice, they were ideally placed to ensure that quality and safety is achieved: 'we have detailed knowledge of medicines, which ones go together, potential adverse effects and such like so we are really competent when it comes to safety' (CP7S3).

Quality and safety have featured centrally throughout the NHS reforms, and have also pervaded debates on prescribing practice particularly in terms of competency and the activities of new prescribers and as a means of containing and constraining prescribing practice. Key concerns for pharmacists were the amount of errors that appeared in the patient drug history charts on admission, nurses adding medications on to the patient chart, and the faxed and verbal 
instructions to the pharmacy all of which are not signed off by a clinician and are clearly in breach of 'Safe Prescribing Practice' as set down by the medical profession.

'We deal with prescribing issues on a regular basis, sometimes a patient's drug history is wrong, so we have to change it, or the nursing staff will change it, but it can be a few days before the clinician is back on the ward which is a problem as the change is not signed off'. (PM2S2).

A report by the Medical Protection agency estimates that "medication errors account for approximately $20 \%$ of all clinical negligence claims against doctors in both primary and secondary care" the cost of which is in the region of $£ 750$ million per annum (Medical Protection, 2016, p.1). The quote above highlights a problem with clinician prescribing (or lack of) in terms of safety which could further justify the extended role of pharmacists into clinical areas and prescribing activities and was seen as a positive step by some respondents. 'Now if we see an error we can make the necessary amendment to the patient's medication regime so there are fewer hold ups with getting their medication to them' (CDP2S2).

Indeed, ensuring quality and safety standards, not just in terms of their own prescribing but also that of the clinicians, particularly the junior clinicians, was concern for all of the respondents.

'We also need to be careful and have controls and checks on our own prescribing the medics also check what we do' (CP1S1).

'I feel it's really important to have clinical pharmacists involved in the prescribing decisions, especially when the clinician is still relatively inexperienced, having us involved helps with safety and improves the quality of patient care' (CDP1S1).

Within the wider political context of the need to ensure quality and safety, the discourses of risk as advocated by Foucault (1977) in his work Discipline and Punish, provide the rationale for self-examination and audit as a professional requirement: 'Keeping abreast of new medicines and professional development is all part of the job. We need to engage in this to provide a good quality service. It's also a professional requirement'(CP5S2). Previous research such as the work of Leicht \& Fennell (1997) on the changing organisational context of professional work and prescribing safety (Bradley \& Nolan, 2008), note that those who refrain from risky behaviour perceive themselves to be more rational and responsible than those who do engage in risky activities, which supports pharmacists' opinions on safe prescribing.

\section{Self-limitation and Interprofessional Working}

The emphasis on developing a culture of safety not only provides a rationale for extending prescribing authority to pharmacists but also opens up explicit discussions on the competence of all prescribers; clinicians included. The focus of safety and the need to be competent promotes a new core professional ideal, particularly for new prescribers and provides a medium of constraint on the desire to prescribe and a level of self-limitation:

'You need be able to justify your prescribing decision, so you need to be aware of the clinical guidelines, protocols and up on evidence-based regimes otherwise we end up with variations in prescribing practice, which is something we are trying to avoid'. (CP3S1) 
As previously noted a common theme that appears within the NHS reforms is the drive towards increased collaboration between healthcare professional groups and interprofessional working teams (DOH, 2000a, 2000b, 2001,2002, 2003, 2004). Within these reforms, the government has mandated a change in the authority to prescribe as exemplified by the introduction of pharmacist prescribing into the system. It can be argued that the function of pharmacy in terms of the level of attention to prescribing detail and dispensing applied provides a valuable safety check on clinical decisions.

'It makes sense that the person with the most pharmacological knowledge should be allowed to prescribe. It not only improves quality and safety but can save money ... and frees up some of the clinician's time ...' (CP6S2).

Additionally, pharmacist's unique competence in terms of their pharmacological expertise and focus on safety can arguably provide a key force on any prescribing jurisdictional claim. Such attributes also exhort the emphasis on self-limitation on practice and the benefits of collaborative teams. It can be further argued that fragmenting the process of prescribing into distinct tasks that pull on each individual's area of competence such drug history taking, clinical examination and diagnosis, and treatment decision and monitoring has the potential to provide improved patient safety and quality of care through a team approach (Bradley \& Nolan, 2008). While the traditional dominating role of clinician prescribing has been taken for granted, the rhetoric of collaborative teams, safety and quality within the managerial logics and NHS reforms has exhorted the idea of each professional group with their respective areas of competence working together to establish a culture of safety and self-imposed limitations. For such teams to be effective cooperation and recognition of professional roles and expertise are essential. However, it was evident that the majority of pharmacist believe that their expertise and ability to take on a prescribing role is both underestimated and underutilised within the hospital setting.

'Clinicians are reluctant to let go, they see it as us encroaching into their area too much ... They put up the argument that we are not sufficiently trained but I think it's more than that they are protecting their own area ... It's not good really, we could help and support them a lot more' (CP1S1).

Indeed, more than half of pharmacist respondents were very conscious that some clinicians did not consider them to have enough knowledge or expertise to safely prescribe, which for some was rather offensive and a cause of tension. It was also noted that some clinicians ignored pharmacist's advice, or overruled their prescribing decisions, thus exercising their dominant position.

'Not all clinicians are on board with us prescribing or making prescribing recommendations some think it's too dangerous for the patients, they're worried that we get it wrong and kill them [patients] (CP7S3).

'Clinicians don't always accept our prescribing decisions or advice, this can be quite frustrating especially as what they prescribe is not necessarily better or more appropriate than what was suggested...sometimes it just feels like they are making a statement' (CP4S2). 
This suggests an element of resistance on the part of clinicians to engage fully with pharmacists prescribing and highlights the continuation of the politics of domination and autonomy in the medical profession and the difficulties of, and barriers to, interprofessional collaboration.

A point that was evident from discussions with the pharmacist respondents concerned their legitimacy to prescribe which, from their point of view, was unquestionable.

'We are trained on the pharmacological effects of medicines on the body, we study physiology and anatomy as part of our training, we discuss medication regimes with patients, so we are in some ways better qualified to prescribe especially compared to some other supplementary prescribers and junior clinicians' (CP8S3).

Indeed, it is evident from the data that pharmacists in general perceive that they have a singular expertise with regards to medicines that is superior to other healthcare professionals including the medical profession.

'We know more about the drug interactions than they do, even before we started to prescribe we had cases where we had to step in and block a medication that was just wrong for the patient given all their other medications and health condition' (CP7S3).

However, the formalising (the act or writing the prescription) of pharmacist prescribing, and the associated responsibility was a concern for virtually all of the pharmacist respondents.

'It makes you think more seriously about the accountability and consequences of what you are prescribing, it's not like telling the clinician what to prescribe and him holding the responsibility, it comes back on me that's a bit scary so getting the right clinical training and keeping abreast of the guidelines is crucial' (CDP2S2).

Despite their overall confidence with pharmacological knowledge another issue that was raised by pharmacist respondents, regarding them taking on a prescribing role, concerned the diagnosis of the patient's condition. This was an area where the pharmacists showed the most reluctance to engage with independent prescribing.

'I am not entirely comfortable with conducting clinical examinations. It's not part of our general [degree programme] training. The diagnosis training, we get is quite short, so I'm not convinced we have the necessary skills to do this competently ... It's one thing the clinician doing the diagnosis and us doing the prescribing but even they [clinicians] get it wrong sometimes so we need to be very careful' (CP8S3).

Another went on to state that:

'At first I tend to casually check with the clinician first for example I'll say Mrs Smith needs medication for eczema I'm going to give her E45 and Betnovate 1 in 4 cream...It gives him a chance to recommend something else but if he says ok then I just order it'. (P3S3). 
It is interesting that even where pharmacists held the authority to prescribe a few (around 10\%) still sought the clinician's approval which is not required within the supplementary or independent prescribing framework and thus indicates a subordinate position. Such views support the theoretical perspective of Friedson's argument that the success of the medical profession to control the healing process was the result of their ability to dominate other healthcare groups (Freidson, 1986). Indeed, a third of pharmacist respondents indicated a preference for the clinicians to continue with the diagnostic role with the pharmacist doing the prescribing and any subsequent drug therapy adjustments. Such a collaborative effort is considered to draw on each individual's expertise and has the potential to improve the overall quality of patient care.

'Clinicians doing the diagnosis and pharmacist doing the prescribing seems the obvious way forward and makes perfect sense, it has the potential to improve the treatment regimens and reduce errors' (PM4S3).

Despite the reservations raised above, in general there was a willingness to engage in professional developments that open up and push the boundaries of professional activity. The expanded role of clinical pharmacists into prescribing activities was perceived by many (three quarters) of the respondents as a professional development opportunity and improvement for their professional status and self-worth:

'Our involvement in clinical activities and prescribing has been good for us, most of the clinicians treat us with more respect now which is great as I feel more respected professionally'. (CP1S1).

'It enables us to exercise our knowledge more broadly and it's beneficial to our professional development. The clinicians listen and engage with us more too'(CP2S1).

While some reservations over the accountability of prescribing was evident, the majority of pharmacists (three quarters) perceived the formalisation of prescribing as an important indicator of acknowledgment of their knowledge and expertise which impacted positively on their professional status and relationship with the medical profession.

'I was initially a bit nervous of prescribing but now I've been doing it for a while I really enjoy it. It's really tested my professionalism and my relationship with the clinicians. It was a bit tense at first, but we have come to appreciate each other's role more I think so we are getting on a lot better' (CP3S1).

In summary, with regard to pharmacist prescribing, mixed views are held as to what constitutes prescribing, with a few reluctantly engaging with it while others embraced the concept fully. On the issue of competency to prescribe, the majority of pharmacist's respondents considered their pharmacological expertise alongside their clinical training placed them in an ideal position to conduct prescribing tasks. However, concerns were raised over safety and accountability of this extended role, particularly when pharmacists were engaging in diagnosis; a procedure that many felt they were undertrained to perform with complete confidence. Despite such reservations, there was general acknowledgement that the changes had positively impacted on the role and status of pharmacists, particularly within the collaborative teams. In the next section the views and perceptions of clinicians on pharmacist prescribing are put forward. 


\section{Clinicians Views on Pharmacist Prescribing:}

As discussed above, the right to prescribe is a traditional hallmark of the medical profession. The opening discussion with clinicians revealed general reservations and concerns over pharmacists taking on formal prescribing activities on a number of grounds. Many clinician respondents did not feel that such a role was suitable for pharmacists as 'they do not have the relevant diagnostic and patient factor skills that are needed to make safe prescribing decisions [Patient factors skills were explained as doctor patient interactions in to getting to know the patient]' (SC3S2). Several respondents also mentioned that the BMA did not approve of this extended role for pharmacists and had portrayed it as 'an irresponsible government initiative that's dangerous for the patients' (C6S3).

Concerns were also raised by half of clinicians over the type and level of prescribing training that was given to pharmacist prescribers as compared to the five or six years of training that the medical profession undertake.

'A big concern is their limited prescribing training. Patient safety has to be ensured. It's fair enough to get them to prescribe within limited areas, but the more chronic illnesses should be kept to the clinician ... They just don't have the diagnostic skills or experience to open this up further' (C7S3).

Another clinician went on to say 'I would not be happy to have a pharmacist making the prescribing decisions for anyone of my family ... It's just not safe ... Diagnosis can be very difficult and complex, so you need a lot of skills in this area which are developed from years of experience ... Pharmacists don't have this experience' (SC4S2).

Diagnosis and prescribing would thus appear to be inextricably linked in the mind of some respondents. Indeed, around half of clinician respondents made recourse to the level of professional training as a justification against pharmacist prescribing, which reflects Abbott's professional archetype that justifies control of a jurisdiction on the grounds of 'first and foremost a right to perform the work as professionals see fit...to exclude other workers [and] to dominate public definitions of the tasks concerned' (Abbott, 1988, p.60). This view however, was not held by all of the clinicians.

'Pharmacists are an integral part of our team and provide us with advice on the most cost-effective treatments and guidance of the best drug combinations to use when we discuss the patient's medications'(C2S1).

'Their input to medication regimens can be really useful' (C5S2).

A feature that regularly appeared within the clinician respondent's discussions was the issue of time. Indeed, all respondents made reference to the amount of time (or lack of) spent with patients. A few respondents seemed genuinely open to pharmacist prescribing and viewed it as a positive development that could free up some time provided they still retained a level of control.

'It frees up some of our time, so we can focus on the more critical patients so in that respect it's good ... But we need to be really careful how this is set up and make sure that they have the right training and support ... and also keep it within the less critical areas' (CD1S1). 
A senior clinician went on to state that the continuous reforms to the service would likely enhance this development further in the future.

'The service is under such financial pressure that we are really overstretched in some areas, so we are becoming more reliant on nursing staff and pharmacists to help take up some of the burden to free us up a bit so that we can spend more time with the chronically ill. It's challenging for them though as they are being expected to do things that they are not always comfortable with. It's also not so good for us as we are losing some of our field to them ... I can't really see this situation getting any better'(CD3S3).

Indeed, the majority of clinician respondents felt that as pharmacists became more involved the kind of prescribing activities undertaken by them would expand and thus might impact on interprofessional working and the work of clinicians and their role as specialists.

\section{Interprofessional Working and Status}

With respect to interprofessional working the data illustrates the importance of the clinicianpharmacist relationship within the hospital setting and indicates as clinical guidelines, protocols and quality standards increase, the power balance between the two groups has the potential to shift in a favourable way for pharmacy as a healthcare profession.

'Giving them prescribing rights has increased their status and their confidence they challenge our decisions sometimes and quote the protocols and guidelines to us. The clinical pharmacist I work with will really stand her ground on some issues'(C5S3).

'The pharmacist that I work with really pushes me to conform to the prescribing guidelines we have some lively debates, but I see her point they are there for a reason, so we should stick to them' (SC2S1)

Pharmacist involvement in prescribing decisions was an area in which several clinicians perceived that the status of pharmacists had improved.

'I think in general this has been a good development for pharmacists. They have a much greater input now into clinical decisions and work alongside us and are part of the decision-making process so for them this has really helped develop their status' (SC3S2).

Indeed, there was a perception among clinicians (just over half) that the esteem and status of the hospital clinician within the last 25 years or so has not only changed vis-à-vis pharmacists but also with the populace. Indeed, patient's perception and trust in the medical profession were recognised as not being as deferential to the notion of the clinician as being the expert as much as it had been in the past.

'Patients expect much more they hear about new treatments and demand them ... some even come to us and tell us what they want and resist no for an answer. In such cases we have to work really hard to convince them that what we are giving them is appropriate and good enough' (C4S2). 
It was however, suggested by threequarters of clinicians that this is one area in which the pharmacists could help. Longer consultations with patients, particularly those with chronic conditions, were considered to be very important, but the time constraints of the clinicians made this difficult to sustain.

'We increasingly rely on non-medical staff [such as pharmacists] to take up the strain and fill in the gaps'

'Pharmacists have more time to spend with the patients, so they can discuss all of the ins and outs of their treatment and reassure the patients that the treatment regime is the right and appropriate one for them' (C4S2).

This would suggest that the clinicians regard the pharmacist as being competent with regard to counselling patients and providing a concordat service. However, this rather contradicts the previous assertion that pharmacist do not possess the necessary patient factor skills to make safe prescribing decisions.

Despite such mixed views over the role of pharmacists in prescribing, a view that featured among most hospital clinicians was that, due to the increasing bureaucratic demand and controls being imposed within the health service, the power of the medical profession in general was declining; perhaps suggesting that the time of the all-powerful hospital clinician may be coming to an end.

'We have significant resource constraints which are a challenge ... the guidelines and protocols are a means to try and achieve good quality of care in a costeffective way, we are expected to follow these as far as possible, but this limits our clinical freedom and ability to treat patients the way we want' (SC4S2).

'We're no longer making all the decisions. Regulation is building, more formal practices are in place so there's a greater need to conform ... What is happening is everything is becoming more transparent through more controls being imposed ... We have to work with and take on board the advice of other healthcare workers ... pharmacists are an important part of the team we rely on them not just for their knowledge of medicines but also for their input into the cost effectiveness of the medication regimes' (C6S3).

The last comment is particularly illuminating as it indicates that the government's relentless attempts to impose managerial logics and workforce reconfigurations into the healthcare system are inescapable. In general, the clinician respondents of this study recognised increasing incursions into their traditional professional domain by members of the pharmacy profession. The reduced dominance and influence as a result of these initiatives has enabled a certain amount of re-stratification within the healthcare system to the benefit of the status of the pharmacy profession.

\section{Conclusion}

This paper considers the impact of attempts to improve efficiency and value for money through managerial logics and workforce reconfigurations that maximise specialist expertise such as pharmacist prescribing in the hospital setting and examines their effect on the interprofessional 
relationships of medical professionals and clinical pharmacists. It has been shown that pharmacy has a long history that overlaps with the medical profession in terms of clinical practice. When considering the changing role of pharmacy from its origins through to present day, in broad terms the responsibilities and duties of hospital pharmacists today compared to the early Spicer-Apothecaries are not significantly different. Indeed, it would appear to have gone full circle from prescribing and dispensing for complete patient care through a period of restriction to preparation, distribution and procurement back to a clinical function in the form of clinical pharmacy which also includes a prescribing role. Such activity echoes the historic function of the apothecary and indicates that the profession is in a state of flux (Anderson, 2001, 2005).

The right to prescribe was identified as one of the key hallmarks of the medical profession and an aspect of their professional jurisdiction (Larson, 1977). The act of prescribing has been shown to be a complex process that involves specific tasks and application of detailed scientific clinical knowledge and experience that lead to a decision point. However, this complexity opens up the possibility for other health professionals such as pharmacists with relevant clinical knowledge to become involved in the process. Pharmacists within secondary care were already involved in clinical activities that influenced or controlled prescribing decisions, before legislative changes to prescribing authority occurred. This combined with the confusion over what constitutes prescribing within the hospital setting (e.g. dose adjustments and the ability of pharmacists to refuse the supply of an inappropriate medicine) resulted in some pharmacist respondents not considering the expansion into prescribing as a major change to their role. Also, in the sites under study, it appears that the development of prescribing activity into the clinical pharmacy function involved a slow process of encroachment and delegation, which supported the perception that increased prescribing authority is not a major change for hospital pharmacists. In this it is clear that the introduction of guidelines, alongside formularies and other policy initiatives has created the necessary bridge to overcome the jurisdictional boundary. However, the expansion of pharmacists into a prescribing role, while supported by government initiatives and the RPSGB has not been a smooth or uncontested transition. Indeed, it was clear from the data that pharmacists were very conscious that some clinicians did not consider them to have enough knowledge or expertise to safely prescribe. It was reported that some clinicians exercised their dominant position by ignoring the pharmacist's advice or overruling their prescribing decisions. For the pharmacists with their strong pharmacological expertise and training, this was a source of significant tension.

It is evident that many medical practitioners retain concerns over the extension of prescribing authority groups such as pharmacists that sit outside the traditional boundaries of the medical profession. Clinician respondents aired concerns about this expanded role on the grounds of safety and limited professional training. The lack of pharmacist's 'patient factor skills' were a particular concern with regards to prescribing safety. However, the counter to this was a recognition amongst clinicians that pharmacists with their specific knowledge base are an integral part of the clinical team that supports clinical decision making. In particular, their input into clinical decisions and prescribing were considered useful in saving clinicians time, freeing them up to spend more time with patients that had more complex needs. Many clinicians regard pharmacists as being competent with regard to counselling patients and providing a concordat service, which is somewhat contradictory to the safety and patient factor skills argument.

The data indicated that most hospital clinicians are feeling the pressure of increasing bureaucratic demands and other controls imposed within the health service. As a result, they are increasingly relying on pharmacists to assume tasks that were previously clearly within the domain of clinicians, a situation that they perceive will continue and which could possibly 
expand and impact further on their professional role. Due to increasing financial and time pressures and the imposition of quality standards and treatment protocols, clinicians frequently succumb to the advice of the clinical pharmacists on prescribing decisions, particularly where it is seen as tactful and non-threatening. There remains significant concern that these initiatives may undermine clinical autonomy and increase the level of bureaucratic control. Perhaps this concern stems from the medical profession's realisation that the act of prescribing is one area in which medical practice can be broken down into distinct technical tasks which can then be delegated to other healthcare professionals such as pharmacists. This is of sociological importance as it indicates that medical practice can be subjected to rationalisation in the same way as other occupations (McKinlay,1982; Ritzer \& Walcak, 1982). As such, it is unsurprising that segments of the medical profession, including some of this study's respondents, view the expansion of prescribing authority to non-medical healthcare groups such as pharmacists with distrust.

Within the sociological literature it is argued that changes to prescribing practice and extending prescribing authority to non-medical personnel such as pharmacists would challenge the professional control, jurisdiction and status of the medical profession. Indeed, it would appear that applying the activity of prescribing as an indicator of medical autonomy, control and professional status held by the medical profession, that this has changed. Furthermore, within the deprofessionalisation thesis, it is argued that the medical profession is losing its cultural authority in terms of prestige and trust as a result of various political changes, increased control, awareness and knowledge of the patients which it serves, and professional role reconfigurations (Harding \& Taylor, 1997). The data suggests a recognition on the clinician's part that they are no longer making all of the decisions, indicating a shift in the level of control. However, in contrast, Allsop (2006) argued that the medical profession, rather than being annihilated have found ways to accommodate such changes and have succeeded in retaining high status relative to other healthcare groups. While this paper does not dispute that opinion, the legislative changes in the 2002 Medicines Control Agency consultation paper and the 2004, Scottish Government report, which extended supplementary prescribing authority to pharmacists and the subsequent 2006, Statutory Instrument, allowing formal independent prescribing by pharmacists would appear to have affected the professional control of this aspect of the clinician's work. While pharmacists did not necessarily consider increased prescribing authority as a major change, the clinician respondents did regard this as a significant change that affected both the pharmacist's status and their interprofessional relationship with the medical profession.

With regard to professional status, Elston, 1993 argues that the function of prescribing at the micro level of patient consultations can be viewed as a sign of professional status. While some reservations over the accountability of prescribing on the part of pharmacists was evident, the majority of pharmacists perceived the formalisation of prescribing as an important indicator of acknowledgment of their knowledge and expertise that impacted positively on their professional status and relationship with the medical profession. Pharmacists indicated that they felt that such activity had enabled them to gain professional status not only within their own professional group but with clinicians as well which is also evident in some of the clinician respondents' comments. This was particularly illuminating as it indicates that the medical profession in general, recognises a loss of prominence and influence which has enabled a certain amount of re-stratification to occur within the healthcare system. Following Haug's (1988) deprofessionalisation argument, pharmacist prescribing represents a loss of a unique quality to the medical profession and thus could be considered as deprofessionalising for the medical profession and a re-professionalisation of pharmacy. The pharmaceuticalisation of 
prescribing, which has been encouraged by the government and the Royal Pharmaceutical Society Great Britain (2002) potentially may be of more importance to medical sociology than its traditional preoccupation with clinical medicine to the exclusion of other occupational groups.

It must be acknowledged, however, that from the data produced for this study it is not possible to interpret the full extent of the impact of hospital pharmacist prescribing on the status of pharmacy as a profession. For instance, it is not clear whether the codification of prescribing knowledge genuinely and enduringly creates a shift in the jurisdictional boundary between medicine and pharmacy, or whether it merely opens up that space to a range of occupational groups such as the nursing profession. It is likely that the state in seeking to reduce the power of one profession would be happy to simply substitute increased power for another. Nevertheless, important issues regarding the interprofessional relationship between pharmacists and clinicians with regards to the prescribing function have been raised. Indeed, the pivotal role that pharmacy can play within medicines management ensures that it will remain as an important aspect of healthcare reforms thus providing scope for further research in this area.

\section{References}

Abbott, A. (1988). The System of Professions, University of Chicago Press, Chicago, IL.

Abernethy, M. A., \& Vagnoni, E. (2004). Power, organisation design and managerial behaviour. Accounting, Organizations and Society, 29:207-225.

Abraham, J. (2009). The Pharmaceutical Industry, the state and the NHS, in Gabe, J. \& Calnan, M., (eds), The Sociology of the Health Service, Routledge, London.

Allan, D. (2001). Narrating nursing jurisdiction: atrocity stories and boundary work, Symbolic Interaction, 24, 75-103.

Allsop, J. (2006). Regaining trust in medicine: professional and state strategies, Current Sociology, 54: 621-636.

Anderson, S.C. (2001). The historical context of pharmacy, in Taylor, K., Harding, G., (eds), Pharmacy Practice, Taylor Francis, London,

Anderson, S. (2005). Making Medicines, Pharmaceutical Press, London.

Audit Commission, (1994). Prescription for change. Technical report, HMSO.

Audit Commission, (2001). A Spoonful of Sugar-Medicines Management in the NHS Hospitals, Audit Commission, London.

Barrett, C.R.B. (1905). History of the Society of Apothecaries, Stock, London.

Blaikie, N. (2000), Designing Social Research, Polity Press, Cambridge.

Bradley, E., \& Nolan, P. (2008). Non-Medical Prescribing: Multidisciplinary Perspectives, Cambridge University Press, Cambridge.

Britten, N. (2001). Prescribing and the defence of clinical autonomy, Sociology of Health and Illness, 23: 478-496. 
Buckley, B.P., Grime, J., \& Blenkinsopp, A. (2006). Inter and intra professional perspectives on non-medical prescribing in an NHS trust. Pharmaceutical Journal, 277: 394-398.

Cassell, C., \& Symon, C. (1994). Qualitative Research in Work Context, in Cassell, C., and Symon, C., (ed), Qualitative Methods in Organisational Research: A Practical Guide, Sage, London, 1-13.

Chua, W.F. (1986). Theoretical Constructions of and by the Real, Accounting Organisations and Society, 583-598.

Claesson, C. (1989). A social pharmacy study of the job development and professional status of pharmacists, Thesis, Uppsala University, Uppsalla.

Connelly, D. (2005). Independent prescribing - pharmacists are given the flexibility of a full formulary, Pharmaceutical Journal, 275: 627-8.

Cooper, R., Anderson, C., Avery, T., \& Bissell, P. (2008). Nurse and Pharmacist supplementary prescribing in the UK - A thematic review of the literature, Health Policy 85(3):277-92.

Corbin, J.M., \& Strauss, A.L. (2008). Basics of Qualitative Research. Sage, Los Angeles, CA.

Court, W.E. (2005). Pharmacy from the ancient world to 1100 AD, in Anderson, S. (ed), Making Medicines: A brief history of pharmacy and pharmaceuticals, Pharmaceutical Press, London.

Denzin, N.K., \& Mettlin, C.J. (1968). Incomplete Professionalisation: the case of pharmacy, Social Forces, 46:375-381.

DOH, (1986). Neighbourhood Nursing: a Focus for Care (Cumberlege Report), DOH, London.

DOH, (1988). The way forward for hospital pharmaceutical services. DOH, London.

DOH, (1989). Report of the Advisory Group on Nurse Prescribing (Crown Report). DOH, London.

DOH, (1999). Review of prescribing, supply and administration of medicines (Final Crown report). DOH, London.

DOH, (2000a). NHS Improvement Plan: A Plan for Investment A plan for Reform, DOH, London.

DOH, (2000b). Pharmacy in the Future: Implementing the NHS Plan, DOH, London.

DOH, (2001). Building a Safer NHS, DOH, London.

DOH, (2002). HR in the NHS Plan. DOH, London.

DOH, (2003). A Vision for Pharmacy in the New NHS, DOH, London.

DOH, (2004). NHS Improvement Plan: Putting People at the Heart of Public Services, DOH, London. 
DOH, (2005). Health Reform in England: Update and Next Steps, DOH, London.

DOH, (2007). Non-Medical Prescribing Programme, Available at: http://webarchive.nationalarchives.gov.uk/+/http://www.dh.gov.uk/en/Healthcare/Medicinespharmacyandindustry/Prescriptions/TheNon-MedicalPrescribingProgramme/Supplementaryprescribing/DH_4123025

DOH, (2008). Pharmacy in England: Building on strengths - delivering the future. DOH, London.

Drennan, V.M., Gabe, J., Halter, M., de Lusignan, S., \& Levenson, R. (2017). Physician associates in primary health care in England: A challenge to professional boundaries?, Social Science \& Medicine, 181:9-16.

Eaton, G., \& Webb, B. (1979). Boundary encroachment: pharmacist in the clinical setting, Sociology of Health and Illness, 1: 69-89

Elston, M.A. (1993). The politics of professional power: medicine in a changing health service in Gabe, J., Calnan, M., \& Bury, M. The Sociology of the Health Service, 58-88, Routledge, London.

Edmunds, J., \& Calnan, M. (2001). The reprofessionalisation of community pharmacy? An exploration of attitudes to extended roles for community pharmacists amongst pharmacists and general practitioners in the United Kingdom, Social Science \& Medicine, 53, 943-955

Foucault, M. (1977). Discipline and Punish, Allen Lane, London.

Freidson, E. (1970). Professional Dominance, Aldine, Chicago, IL.

Freidson, E. (1986). Professional Powers, University of Chicago Press, Chicago.

Freidson, E. (1988). Profession of Medicine: A Study in the Sociology of Applied Knowledge, University of Chicago Press, Chicago.

Freidson, E. (1994). Professionalism Reborn. Theory, Prophecy and Policy, Polity Press, Cambridge.

Freidson, E., \& Lorber, J. (1972). Medical Men and their Work, Aldine, Chicago.

General Pharmaceutical Council, (2018). Pharmacist Independent Prescriber, Available at: https://www.pharmacyregulation.org/education/pharmacist-independent-prescriber

Gray, A., \& Harrison, S. (2004). Governing Medicine: Theory and Practice, Open University Press, Buckingham.

Harding, G.D., \& Taylor, K. (1997). Responding to change: the case of community pharmacy in Great Britain, Sociology of Health and Illness, 19:547-560.

Harrison, S. (2003). Health policy, in Kogan, M., and, Hawksworth, M., (eds), The Routledge Encyclopaedia of Government and Politics, second edition, Routledge, London. 
Harrison, S., \& Checkland, K. (2009). Evidence-based practice in UK health policy, in Gabe, J. and Calnan, M., The New Sociology of the Health Service, p 121-142, Routledge, London.

Harrison, S., \& McDonald, R. (2003). Science, consumerism and bureaucracy: new legitimations of medical professionalism, International Journal of Public Sector Management, 16 (2): 110-121.

Haug, M. R. (1988). A re-examination of the hypothesis of physician deprofessionalisation, Millbank Quarterly, 66 (suppl. 2).

Holloway, S.W.F., Jewson, N.D., \& Mason, D.J. (1986). "Reprofessionalisation” or "occupational imperialism"? Some reflections on pharmacy in Britain, Social Science and Medicine, 23:323-332.

Homan, P.G. (2005). The Development of Community Pharmacy, in Anderson, S. (ed), Making Medicines: A brief history of pharmacy and pharmaceuticals, Pharmaceutical Press, London.

Jackson, W.J., Paterson, A.S., Pong C.K.M., \& Scarparo S. (2014). Cash Limits, Hospital Prescribing and Shrinking Medical Jurisdiction, Financial Accountability and Management, 30 (4): 403-429

Jamous, H., \& Peloille, B. (1970). Changes in the French university hospital system, in Jackson, J, A. (ed), Professions \& Professionalisim, Cambridge University Press, Cambridge.

Kislov, R. (2014). Boundary discontinuity in a constellation of interconnected practices. Public Administration, 92(2): 307-323

Larson, M.S. (1977). The rise of professionalism: A sociological analysis. Berkley: University of California Press.

Leicht, K.T., \& Fennell, M.L. (1997). "The changing organizational context of professional work", Annual Review of Sociology, 23:215-31.

MacDonald, K.M. (1995). The Sociology of the Professions, Sage, London.

McDonald, R., Cheraghi-Sohi, S., Sanders, C., \& Ashcroft, D. (2010). Professional status in a changing world: The case of medicines use reviews in English community pharmacy, Social Science \& Medicine, 71, 451-458.

McKinlay, J.B. (1982). Toward the proletarianisation of physicians, in Derber, C. (Ed.), Professionals as Workers: Mental Labor in Advanced Capitalism, G.K. Hall, Boston, MA, 37-62.

Martin, G.P., Currie, G., \& Finn, R. (2009). Reconfiguring or reproducing intra-professional Boundaries? Specialist expertise, generalist knowledge and the 'modernization' of the medical workforce. Social Science and Medicine, 68:1191-1198.

Medical Protection, (2016). Safe Prescribing Scotland Factsheets, Available at: http://www.medicalprotection.org/uk/resources/scotland-factsheets/scotland-factsheets/uksco-safe-prescribing 
Medicines Control Agency, (2002). Proposals for Supplementary Prescribing and Pharmacists and Proposed Amendments to the Prescription Only Medicines (Human Use) Order 1997. Consultation paper MLX284, Medicines Control Agency, London.

Mesler, M.A. (1991). Boundary encroachment and task delegation: clinical pharmacists on the medical team, Sociology of Health and Illness, 13: 310-331.

NHS Executive, (1999). Clinical Governance, HSC 1999/065, NHS Executive, Leeds.

Paterson, A.S., Leung D., Jackson W., Macintosh, R., \& O'Gorman, K. Eds. (2016). Research Methods for Accounting and Finance, Goodfellows, Oxford. Anonymous

Poynter, F.N.L. (1965). The Evolution of Pharmacy in Britain, Pitman Medical, London.

Ritzer, G., \& Walcak, D. (1982). Rationalisation and deprofessionalisation of physicians, Social Forces, 67:1-22.

Royal Pharmaceutical Society of Great Britain, (1986). Pharmacy in a New Age, The Society: London.

Royal Pharmaceutical Society of Great Britain, (2002). Pharmacists Prescribing Task Group: Supplementary prescribing by pharmacists (first report), The Society: London

Royal Pharmaceutical Society of Great Britain, (2009). Principles of Safe and Appropriate Production of Medicine Administration Charts, The Society: London, Available at: https://www.rpharms.com/Portals/0/RPS\%20document\%20library/Open\%20access/Hub/production-medicine-administration-charts.pdf

Scottish Government, (2004). Supplementary Prescribing Pharmacist Practitioners - A Guide for Implementation within NHS Scotland, Available at: http://www.gov.scot/Publica$\underline{\text { tions/2004/06/19514/39165 }}$

Statutory Instruments, (2006). No915, Medicines, The Medicines for Human Use (Prescribing) (Miscellaneous Amendments) Order 2006, The UK Stationary Office.

Schultz, A. (1967). The Phenomenology of the Social World, Northwestern University Press, Evanston.

Thomas, J., \& Harden, A. (2007). Methods for the thematic synthesis of qualitative research in systematic reviews, Available at: http://eprints.ncrm.ac.uk/468/1/1007_JTAHthematic_synthesis.pdf

Trease, G.E. (1964). Pharmacy in History, Bailliere, Tindell and Cox, London.

Turner, B.S. (1995). Medical Power and Social Knowledge, Sage, London.

Weiss, M., \& Fitzpatrick, R. (1997). Challenges to medicine: the case of prescribing, Sociology of Health and Illness, 19: 297-327.

Weiss \& Sutton, (2009). The changing nature of prescribing, Sociology of Health and Illness, 31: 406-421. 
The Worshipful Society of Apothecaries of London, Available at: http://www.apothecaries.org/charity/history/our-history 


\begin{tabular}{|c|c|c|}
\hline Appendix 1: Participant Codes & Date & Duration \\
\hline Clinical Directorate Pharmacist (CDP1 Site 1) & Nov-11 & 50 mins \\
\hline Clinical Directorate Pharmacist (CDP2 Site 2) & Feb-15 & 45 mins \\
\hline Clinical Directorate Pharmacist (CDP3 Site 3) & Mar-16 & 45 mins \\
\hline Pharmacy Manager (PM1 Site 1) & Feb-12 & 40 mins \\
\hline Pharmacy Manager (PM2 Site 2) & Nov-11 & 65 mins \\
\hline Pharmacy Manager (PM 3 Site 2) & Feb-15 & 50 mins \\
\hline Pharmacy Manager (PM4 Site 3) & Feb-12 & 50 mins \\
\hline Pharmacy Manager (PM5 Site 3) & Mar-16 & 55 mins \\
\hline Clinical Pharmacist (CP1 Site 1) & Feb-12 & 35 mins \\
\hline Clinical Pharmacist (CP2 Site 1) & Feb-15 & 55 mins \\
\hline Clinical Pharmacist (CP3 Site 1) & Mar-16 & 45 mins \\
\hline Clinical Pharmacist (CP4 Site 2) & Feb-12 & 45 mins \\
\hline Clinical Pharmacist (CP5 Site 2) & Feb-15 & 45 mins \\
\hline Clinical Pharmacist (CP6 Site 2) & Feb-15 & 35 mins \\
\hline Clinical Pharmacist (CP7 Site 3) & Nov-11 & 50 mins \\
\hline Clinical Pharmacist (CP8 Site 3) & Mar-16 & 40 mins \\
\hline Clinical Pharmacist (CP9 Site 3) & Mar-16 & 40 mins \\
\hline Pharmacist (P1 Site 1) & Feb-12 & 35 mins \\
\hline Pharmacist (P2 Site 2) & Feb-15 & $40 \mathrm{mins}$ \\
\hline Pharmacist (P3 Site 3) & Mar-16 & 40 mins \\
\hline Clinical Director (CD1 Site 1) & Nov-11 & 60 mins \\
\hline Clinical Director (CD2 Site 2) & Feb-12 & 55 mins \\
\hline Clinical Director (CD3 Site 3) & Mar-16 & 55 mins \\
\hline Senior Clinician (SC1 Site 1) & Feb-12 & 60 mins \\
\hline Senior Clinician (SC2 Site 1) & Feb-15 & 35 mins \\
\hline Senior Clinician (SC3 Site 2) & Nov-11 & 55 mins \\
\hline Senior Clinician (SC4 Site 2) & Mar-16 & $45 \mathrm{mins}$ \\
\hline Senior Clinician (SC5 Site 3) & Feb-15 & 30 mins \\
\hline Senior Clinician (SC6 Site 3) & Mar-16 & 60 mins \\
\hline Clinician (C1 Site 1) & Feb-12 & 55 mins \\
\hline Clinician (C2 Site 1) & Feb-15 & 40 mins \\
\hline Clinician (C3 Site 1) & Mar-16 & 38 mins \\
\hline Clinician (C4 Site 2) & Feb-12 & 30 mins \\
\hline Clinician (C5 Site 2) & Feb-15 & 30 mins \\
\hline Clinician (C6 Site 3) & Nov-11 & 55 mins \\
\hline Clinician (C7 Site 3) & Mar-16 & 65 mins \\
\hline
\end{tabular}

\footnotetext{
i Nurse prescribing is outwith the scope of this paper, however the following documents are useful to those wishing to investigate nurse prescribing more fully. Neighbourhood nursing: a focus for care (Cumberlege Report, 1986) recommended that community nurses should be able to prescribe, as part of their everyday nursing care, from a limited list of items. The Crown Report (1989) endorsed nurse prescribing and highlighted the circumstances in which it could occur, and a successful private members bill led to the primary legislation (Medicinal Products: Prescription by Nurses etc. Act 1992) that provided the power for nurses to prescribe available at: https://www.legislation.gov.uk/ukpga/1992/28/pdfs/ukpga_19920028_en.pdf. https://www.publichealth.hscni.net/directorate-nursing-and-allied-health-professions/nursing/nurse-prescribing ii It is important to note the substantial differential in the pay scales of clinicians and pharmacists at this point.
} 
iii Area Prescribing and Medicines Management Committees were established for all NHS Trusts.

iv Each of these committees are made up of lead clinicians and pharmacists from both primary and secondary care, commissioning medicines advisors, patient/ pubic representatives and other relevant healthcare support staff.

${ }^{\vee}$ Prescribing guidelines and protocols are recommendations for good practice based on the clinical and costeffectiveness information for people involved in governing, commissioning, prescribing and making decisions about medicines.

vi Also see Department of Health, 2000a \& 2000b, 2003, 2007, 2008.

vii The I/T ratio was coined by Jamous and Peloille (1970). It refers to an increase in the proportion of elements within medical work that can be clearly specified, with a corresponding reduction in the proportion that must be left to the judgement of the clinician. 